hep-ph/9901201

Saclay/SPhT-T98/144

\title{
All-Order Collinear Behavior in Gauge Theories
}

\author{
David A. Kosower \\ Service de Physique Théorique \\ Centre d'Etudes de Saclay \\ F-91191 Gif-sur-Yvette cedex, France \\ kosower@spht.saclay.cea.fr
}

\begin{abstract}
I give a proof, using the unitarity-based method, of the collinear factorization of the leading-color contribution to gauge-theory amplitudes. The proof also provides a concrete formula which can be used to compute the associated splitting amplitudes.
\end{abstract}

Submitted to Nuclear Physics B

${ }^{\natural}$ Laboratory of the Direction des Sciences de la Matière of the Commissariat à l'Energie Atomique of France. 


\section{Introduction}

Gauge-theory amplitudes have simple and universal features in various singular limits. In the collinear limit, when the momenta of two massless particles become proportional $\left(k_{a} \rightarrow z\left(k_{a}+k_{b}\right)\right.$, $\left.k_{b} \rightarrow(1-z)\left(k_{a}+k_{b}\right)\right)$, we may summarize these factorization properties in a set of universal splitting amplitudes. The squares of the tree-level collinear splitting amplitudes, summed over helicities, yield the Altarelli-Parisi kernels [1,2]. Their integrals play an important role in the infrared cancellations that are needed for calculations of physical quantities at next-to-leading order in perturbative quantum chromodynamics $[3,4,5,6]$.

Beyond leading order, splitting amplitudes also have relatively simple forms. Their explicit forms at one loop $[7,8]$ were extracted by taking the collinear limit in various five-point one-loop amplitudes. They are useful as complements to the unitarity-based method of computing one-loop amplitudes $[9,10]$, and will play a role in the cancellation of infrared divergences in next-to-nextto-leading order calculations.

Bern and Chalmers [11] have previously given a proof of collinear factorization at one loop. It has the virtue of being quite general, and not limited to gauge theories. It is unclear, however, how to generalize their proof beyond one loop. Furthermore, even at one loop it provides neither a simple understanding of the structure of the splitting amplitudes, nor a simple method of computing them. The purpose of this paper is to provide an independent proof of collinear factorization which generalizes readily to higher loops. It will also provide a simple and concrete formula for computing the splitting amplitudes at one and two loops. The proof is an application of the unitarity-based method for computing loop amplitudes developed by Bern, Dixon, Dunbar, and the author $[9,10]$.

\section{Order of Limits}

The properties of non-Abelian gauge-theory amplitudes in the collinear limit are easiest to express in the context of a color decomposition [12]. For tree-level all-gluon amplitudes in an $S U(N)$ gauge theory the color decomposition has the form,

$$
\mathcal{A}_{n}^{\text {tree }}\left(\left\{k_{i}, \lambda_{i}, a_{i}\right\}\right)=\sum_{\sigma \in S_{n} / Z_{n}} \operatorname{Tr}\left(T^{a_{\sigma(1)}} \cdots T^{a_{\sigma(n)}}\right) A_{n}^{\text {tree }}\left(\sigma\left(1^{\lambda_{1}}, \ldots, n^{\lambda_{n}}\right)\right)
$$

where $S_{n} / Z_{n}$ is the group of non-cyclic permutations on $n$ symbols, and $j^{\lambda_{j}}$ denotes the $j$-th momentum and helicity. As is by now standard, I use the normalization $\operatorname{Tr}\left(T^{a} T^{b}\right)=\delta^{a b}$, and I 
suppress powers of the coupling constant. One can write analogous formulæ for amplitudes with quark-antiquark pairs or uncolored external lines. The color-ordered or partial amplitude $A_{n}$ is gauge invariant.

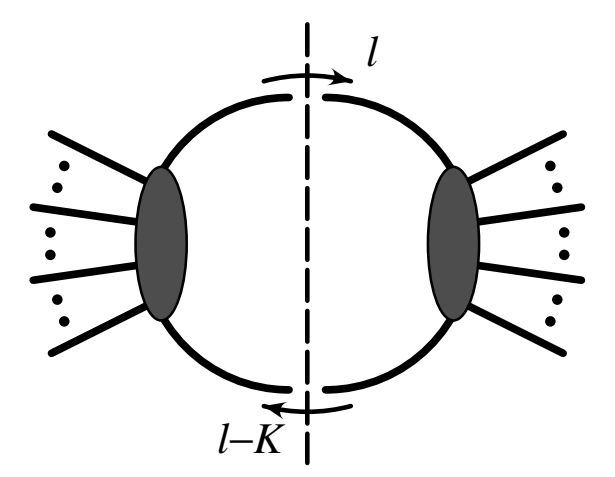

Figure 1. The discontinuity of a loop amplitude expressed as a product of tree amplitudes.

In the cut-based approach to one-loop calculations, we have the following basic equation,

$$
A^{1 \text { loop }}=\sum_{\text {cuts } K^{2}} \int \frac{d^{4-2 \epsilon} \ell}{(2 \pi)^{4-2 \epsilon}} \frac{i}{\ell^{2}} A_{\text {left }}^{\text {tree }} \frac{i}{(\ell-K)^{2}} A_{\text {right }}^{\text {tree }}
$$

where the loop integral is promoted from the integral over phase space, and the sum over all cuts is understood to count only once integral functions that have cuts in multiple channels. The righthand side is depicted in fig. 1. (I will leave the sum over all intermediate particle types and physical polarizations implicit in the cut expression. In the four-dimensional helicity scheme $[13,14]$, these would be the positive and negative helicity states; in the conventional dimensional regularization scheme [15], it would include ' $\epsilon$ ' helicities as well.)

In general, we are interested only in the resulting terms through finite order in the dimensional regularization parameter $\epsilon$. Reconstructing the amplitude by summing over the cuts does not however commute with expanding around $\epsilon=0$; only if all cuts satsify a power-counting criterion can the full amplitude, to order $\epsilon^{0}$, be reconstructed from the $D=4$ tree amplitudes on either side of the cut. Otherwise, we must keep the cuts to higher orders in $\epsilon$ (or use other tricks) in order to obtain the finite rational terms in the amplitude.

The singular limit in which we are interested poses additional interchange-of-limits problems. The limit in which $k_{a} \| k_{b}$, or more generally in which a single invariant $s_{a b}=\left(k_{a}+k_{b}\right)^{2}$ of two color-adjacent legs vanishes while all other invariants stay (roughly) fixed, fails to commute with the expansion around $\epsilon=0$, as can be seen in the example,

$$
\lim _{s \rightarrow 0} \frac{s^{-\epsilon}}{\epsilon^{2}}=0 \neq \lim _{s \rightarrow 0} \frac{1}{\epsilon^{2}}-\frac{\ln s}{\epsilon}+\frac{\ln ^{2} s}{2} .
$$


(As is conventional in investigating infrared divergences, I will take $\epsilon<0$.) This interchange-oflimits issue in specific integral functions is addressed by the Bern-Chalmers discontinuity functions [11].

In what order should we take the singular and four-dimensional limits? It might seem more natural to take the $\epsilon \rightarrow 0$ limit first, but this is not the best choice. The problem is precisely that in this order, we lose control not only of divergent terms but also of finite terms in the integrals (the $\ln ^{2} s$ above). Keeping them under control would mean keeping track of all finite terms in integrals, a rather formidable task. On the other hand, if we take the singular limit first, then all one-loop integrals with more than one external invariant (i.e. all but one-mass triangles and bubbles) are in fact finite in any singular limit. This may seem bizarre - what about the st in the denominator of the one-mass box, for example? — but is easily seen to be true using the Feynman parameter representation. (The resulting collinear-limit integral may, of course, be more singular in the later $\epsilon \rightarrow 0$ limit, but that is a later concern.) Indeed, it is instructive to consider the $s, t \rightarrow 0$ limit of the reduced one-mass box [16]; what naively looks to be uncontrolled growth the standard $\epsilon \rightarrow 0$ expansion,

$$
\begin{aligned}
I_{4}^{1 \mathrm{~m}}\left(s, t, m^{2}\right) & =\frac{r_{\Gamma}}{s t}\left\{\frac{2}{\epsilon^{2}}\left[1+\epsilon \ln \left(\frac{\mu^{2}}{-s}\right)+\epsilon \ln \left(\frac{\mu^{2}}{-t}\right)-\epsilon \ln \left(\frac{\mu^{2}}{-m^{2}}\right)\right]\right. \\
& +\ln ^{2}\left(\frac{\mu^{2}}{-s}\right)+\ln ^{2}\left(\frac{\mu^{2}}{-t}\right)+\ln ^{2}\left(\frac{\mu^{2}}{-m^{2}}\right) \\
& \left.-2 \operatorname{Li}_{2}\left(1-\frac{m^{2}}{s}\right)-2 \operatorname{Li}_{2}\left(1-\frac{m^{2}}{t}\right)-\ln ^{2}\left(\frac{s}{t}\right)-\frac{\pi^{2}}{3}\right\}+\mathcal{O}(\epsilon),
\end{aligned}
$$

(with $\left.r_{\Gamma} \equiv \Gamma(1+\epsilon) \Gamma^{2}(1-\epsilon) / \Gamma(1-2 \epsilon)\right)$ turns out to be quite well behaved (after analytic continuation) if we go back to the Feynman parameter representation,

$$
\begin{aligned}
\left(\mu^{2}\right)^{\epsilon} \Gamma(2+\epsilon) \int_{0}^{1} d^{4} a \frac{\delta\left(1-\sum_{i} a_{i}\right)}{\left[-s a_{1} a_{3}-t a_{2} a_{4}-m^{2} a_{4} a_{1}\right]^{2+\epsilon}} & \longrightarrow\left(\mu^{2}\right)^{\epsilon} \Gamma(2+\epsilon) \int_{0}^{1} d^{4} a \frac{\delta\left(1-\sum_{i} a_{i}\right)}{\left[-m^{2} a_{4} a_{1}\right]^{2+\epsilon}} \\
& =-2 r_{\Gamma}\left(\mu^{2}\right)^{\epsilon}\left(-m^{2}\right)^{-2-\epsilon}
\end{aligned}
$$

The analytic continuation involved, however, is not appropriate for us, as it amounts to subtracting poles in $s$ and $t$. (The scale $\mu$ is introduced by dimensional regularization and would eventually become the $\overline{\mathrm{MS}}$ renormalization scale.)

We do want to pick up such poles, so the appropriate limit for our purposes is given by expanding the integrated form, leaving epsilonic powers alone, and taking care to drop only terms suppressed by integer powers of $s / m^{2}$ or $t / m^{2}$. 
Using the form of $I_{4}^{1 \mathrm{~m}}$ expressed in terms of hypergeometric functions,

$$
\begin{aligned}
\frac{2 r_{\Gamma}}{\epsilon^{2} s t} & {\left[\left(\frac{s t}{\mu^{2}\left(t-m^{2}\right)}\right)^{-\epsilon}{ }_{2} F_{1}\left(-\epsilon,-\epsilon ; 1-\epsilon ; \frac{s+t-m^{2}}{t-m^{2}}\right)\right.} \\
+ & \left(\frac{s t}{s-m^{2}}\right)^{-\epsilon}{ }_{2} F_{1}\left(-\epsilon,-\epsilon ; 1-\epsilon ; \frac{s+t-m^{2}}{\mu^{2}\left(s-m^{2}\right)}\right) \\
& \left.-\left(\frac{s t m^{2}}{\left(s+t-m^{2}\right) m^{2}-s t}\right)^{-\epsilon}{ }_{2} F_{1}\left(-\epsilon,-\epsilon ; 1-\epsilon ; \frac{\left(s+t-m^{2}\right) m^{2}}{\mu^{2}\left[\left(s+t-m^{2}\right) m^{2}-s t\right]}\right)\right],
\end{aligned}
$$

dropping terms suppressed by integer powers of (say) $s / m^{2}$, and only then expanding in $\epsilon$, we find

$$
\begin{aligned}
& \frac{2 r_{\Gamma}}{\epsilon^{2}}\left(\frac{s t}{-m^{2} \mu^{2}}\right)^{-\epsilon}{ }_{2} F_{1}(-\epsilon,-\epsilon ; 1-\epsilon ; 1)=\frac{2 r_{\Gamma}}{\epsilon^{2}}\left(\frac{s t}{-m^{2} \mu^{2}}\right)^{-\epsilon} \Gamma(1-\epsilon) \Gamma(1+\epsilon) \\
& =\frac{2 r_{\Gamma}}{\epsilon^{2}}\left(\frac{(-s)(-t)}{-m^{2} \mu^{2}}\right)^{-\epsilon}+\frac{\pi^{2}}{3}+\mathcal{O}(\epsilon)
\end{aligned}
$$

which is well-behaved in the singular limit. We should thus take the singular limit first, and only then the four-dimensional one. In the following discussion, unless otherwise stated, I will then keep amplitudes and cut expressions to all orders in $\epsilon$, unexpanded in a series.

Of course, there is no free lunch. The discrepancy between eqn. (2.5) and (2.7) hints at the problem that naive manipulations with the Feynman-parameter representation dispose of terms like $s^{-\epsilon}$, which we really want to keep. If we are considering a cut in one channel, say $s_{1}$, and the resulting cut is proportional to $s_{2}^{-\epsilon}$, where $s_{2} \rightarrow 0$ in the desired singular limit, then it would appear at first glance that this contribution will disappear quietly, and we won't pick it up. (Of course, if the $s_{2}^{-\epsilon}$ does not show up as part of an integral function containing a cut in the $s_{1}$ channel, but merely as a separate function from the integral reductions, then it should be dropped anyway because the cut in the $s_{1}$ channel necessarily has an ambiguity of that form.) However, this contribution will also have a cut in the $s_{2}$ channel, and we can expect to pick it up there. We will therefore have to be very careful about taking the limit of the cut in the singular channel. This subtlety would also mean in considering singular limits with more than one singular invariant, that we would have to take cuts in multiple channels simultaneously, in order to pick up contributions proportional to $s_{1}^{-\epsilon} s_{2}^{-\epsilon}$.

While we cannot take the singular limit before computing the cut for the cut in the singular channel itself, what about other channels? In general, an inverse integer power of the singular invariant must be supplied by the amplitudes on either side of the cut, or else by the loop integral whose discontinuity is given by the cut. (The helicity algebra eventually softens this singularity to a square-root from a full pole.) If the inverse integer power is supplied by an amplitude on either side of the cut, we can clearly take the singular limit before considering the cut. The same 
turns out to be true (except in the singular channel, as discussed above) even if the inverse integer power arises from the loop integral itself. Because we are working to all orders in $\epsilon$, the integral will necessarily have a cut in the singular channel. Thus whatever mistake we would be making by taking the limit naively in some other channel will be fixed up when we consider the cut in the singular channel itself. This implies, in particular, that cuts in which the collinear momenta are on opposite sides of the cut can be dropped. Such configurations do give contributions in the collinear limit, for example in the form of the hard two-mass box integral; but this contribution can be picked up in the singular channel.

\section{Collinear Factorization at One Loop}

At one loop, all amplitudes can be written as sums of leading-color amplitudes*. It is thus sufficient to consider the collinear limit of leading-color amplitudes in order to characterize the complete collinear behavior of a one-loop amplitude. Furthermore, the amplitude is finite in the limit unless the two collinear legs are (color-) adjacent, so restrict attention to this case.

The amplitude can be reconstructed completely from the knowledge of all cuts; because we are working to all orders in $\epsilon$, there is no possible rational ambiguity. If we can determine the collinear limits of all cuts, we will then have determined the collinear limit of the amplitude.

Consider first a generic cut, in which the two collinear legs $a$ and $b$ are a proper subset of the legs on one side of the cut, and label the legs on the other side from $c$ through $d$. The cut in this channel, $t_{c \ldots d}=\left(k_{c}+\cdots+k_{d}\right)^{2}$, is then

$$
\begin{aligned}
& \left.A_{n}^{1-\text { loop }}(1, \ldots, a, b, \ldots, n)\right|_{t_{c \cdots d} \text { cut }}= \\
& \quad \int d \operatorname{LIPS}^{4-2 \epsilon}\left(\ell_{1},-\ell_{2}\right) A_{n-m+2}^{\text {tree }}\left(\ell_{1}, c, \ldots, d,-\ell_{2}\right) A_{m+2}^{\text {tree }}\left(\ell_{2}, d+1, \ldots, a, b, \ldots, c-1,-\ell_{1}\right)
\end{aligned}
$$

To obtain the collinear limits of this expression, we must make use of the tree-level factorization,

$$
A_{n}^{\text {tree }}\left(1, \ldots, a^{\lambda_{a}}, b^{\lambda_{b}}, \ldots, n\right) \stackrel{k_{a} \cdot k_{b} \rightarrow 0}{\longrightarrow} \sum_{\text {ph. pol. } \sigma} \operatorname{Split}_{-\sigma}^{\text {tree }}\left(a^{\lambda_{a}}, b^{\lambda_{b}}\right) A_{n-1}^{\text {tree }}\left(1, \ldots,(a+b)^{\sigma}, \ldots, n\right)
$$

where Split ${ }^{\text {tree }}$ is the usual tree splitting amplitude, the notation ' $a+b$ ' means $k_{a}+k_{b}$, and where the notation 'ph. pol.' indicates a sum over physical polarizations only. $\operatorname{Split}^{\text {tree }}(a, b)$ goes as $s_{a b}^{-1 / 2}$; terms not singular in the limit are omitted in this factorization. This factorization is depicted schematically in fig. 1 .

\footnotetext{
* Or primitive amplitudes, when some of the external legs are fermions.
} 
This form may be derived from either the Berends-Giele recurrence relations [17], or else [18] from the Koba-Nielsen open-string amplitude [19]. Either derivation shows that this factorization holds only for on-shell (that is, physically polarized) legs $a, b$, but in arbitrary dimension. The following arguments will thus go through equally well in the four-dimensional helicity scheme, the conventional dimensional regularization scheme, or the original 't Hooft-Veltman scheme.

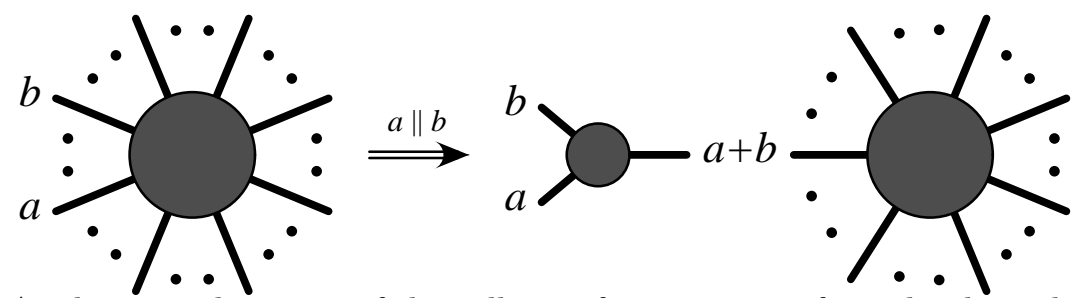

Figure 1. A schematic depiction of the collinear factorization of tree-level amplitudes, with the amplitudes labelled clockwise.

The tree splitting amplitude is given by the appropriate limit of the three-point Berends-Giele current,

$\operatorname{Split}_{\sigma}^{\mathrm{tree}}\left(a^{\lambda_{a}}, b^{\lambda_{b}}\right)=\frac{1}{\sqrt{2} s_{a b}}\left[\varepsilon_{a}^{\left(\lambda_{a}\right)} \cdot \varepsilon_{b}^{\left(\lambda_{b}\right)}\left(k_{b}-k_{a}\right) \cdot \varepsilon_{-\Sigma}^{(\sigma)}+2 k_{a} \cdot \varepsilon_{b}^{\left(\lambda_{b}\right)} \varepsilon_{a}^{\left(\lambda_{a}\right)} \cdot \varepsilon_{-\Sigma}^{(\sigma)}-2 k_{b} \cdot \varepsilon_{a}^{\left(\lambda_{a}\right)} \varepsilon_{b}^{\left(\lambda_{b}\right)} \cdot \varepsilon_{-\Sigma}^{(\sigma)}\right]$

where $\Sigma$ denotes the fused leg, $k_{\Sigma}=k_{a}+k_{b}$.

In the limit, eqn. (3.1) then yields,

$$
\begin{aligned}
& \quad \sum_{\text {ph. pol. } \sigma} \operatorname{Split}_{-\sigma}^{\text {tree }}\left(a^{\lambda_{a}}, b^{\lambda_{b}}\right) \int d \operatorname{LIPS}^{4-2 \epsilon}\left(\ell_{1},-\ell_{2}\right) \\
& \quad \times A_{n-m+2}^{\text {tree }}\left(\ell_{1}, c, \ldots, d,-\ell_{2}\right) A_{m+1}^{\text {tree }}\left(\ell_{2}, d+1, \ldots,(a+b)^{\sigma}, \ldots, c-1,-\ell_{1}\right) \\
& =\left.\sum_{\text {ph. pol. } \sigma} \operatorname{Split}_{-\sigma}^{\text {tree }}\left(a^{\lambda_{a}}, b^{\lambda_{b}}\right) A_{n-1}^{1-\text { loop }}\left(1, \ldots,(a+b)^{\sigma}, \ldots, n\right)\right|_{t_{c \cdots d} \text { cut }} \cdot
\end{aligned}
$$

As noted in section 2, we need not consider cuts where the momenta are on opposite sides of the cut (in which case they are both necessarily adjacent to it). The above derivation breaks down, as expected, if $a$ and $b$ are the only legs on one side of the cut; but all contributions except those detectable in the singular channel take the form presented in eqn. (3.4). This leaves us with the singular channel, which I consider next. 


\section{The Singular Channel}

The cut in the singular channel has the following form,

$$
\begin{aligned}
& \left.A_{n}^{1 \text {-loop }}(1, \ldots, a, b, \ldots, n)\right|_{s_{a b} \text { cut }}= \\
& \quad \sum_{\text {ph. pol. } \lambda_{1,2}} \int d \operatorname{LIPS}^{4-2 \epsilon}\left(\ell_{1}, \ell_{2}\right) A_{n}^{\text {tree }}\left(-\ell_{1}^{-\lambda_{1}}, b+1, \ldots, a-1,-\ell_{2}^{-\lambda_{2}}\right) A_{4}^{\text {tree }}\left(\ell_{2}^{\lambda_{2}}, a, b, \ell_{1}^{\lambda_{1}}\right) .
\end{aligned}
$$

(I have relabelled $\ell_{1} \rightarrow-\ell_{1}$ in this equation.) We already know we must treat it carefully, in particular we are not allowed to take the singular limit before performing the cut integral. It is nonetheless reassuring to see that the expression itself raises several warning flags, independent of the discussion in section 2. The cut integral is somewhat peculiar, because the volume of phase space vanishes in the singular limit. Furthermore, the factorization we used in the previous section fails. We cannot factorize the four-point amplitude into splitting amplitudes times three-point amplitudes, because the three-point amplitude vanishes on-shell. (Note, incidentally, that this does not mean that the four-point amplitude is not singular in this limit; on the contrary, it is more singular, having a full pole rather than just a square-root singularity. This is of course just the usual forward-scattering singularity of a gauge theory.)

The other special feature of this cut is what allows us to simplify this expression. In this cut, momentum conservation conservation forces $s_{\ell_{1} \ell_{2}} \longrightarrow 0$ in the limit. This in turn allows us to use the tree-level factorization (3.2) on the amplitude on the left-hand side of the cut (4.1),

$$
\begin{aligned}
& A_{n}^{\text {tree }}\left(-\ell_{1}^{-\lambda_{1}}, b+1, \ldots, a-1,-\ell_{2}^{-\lambda_{2}}\right) \stackrel{s_{a b} \rightarrow 0}{\longrightarrow} \\
& \sum_{\text {ph. pol. } \sigma} \operatorname{Split}_{-\sigma}^{\text {tree }}\left(-\ell_{2}^{-\lambda_{2}},-\ell_{1}^{-\lambda_{1}}\right) A_{n-1}^{\text {tree }}\left(\left(-\ell_{1}-\ell_{2}\right)^{\sigma}, b+1, \ldots, a-1\right) \\
&= \sum_{\text {ph. pol. } \sigma} \operatorname{Split}_{-\sigma}^{\text {tree }}\left(-\ell_{2}^{-\lambda_{2}},-\ell_{1}^{-\lambda_{1}}\right) A_{n-1}^{\text {tree }}\left((a+b)^{\sigma}, b+1, \ldots, a-1\right) .
\end{aligned}
$$

Note that the only remaining dependence on the cut momenta is in the tree-level splitting function.

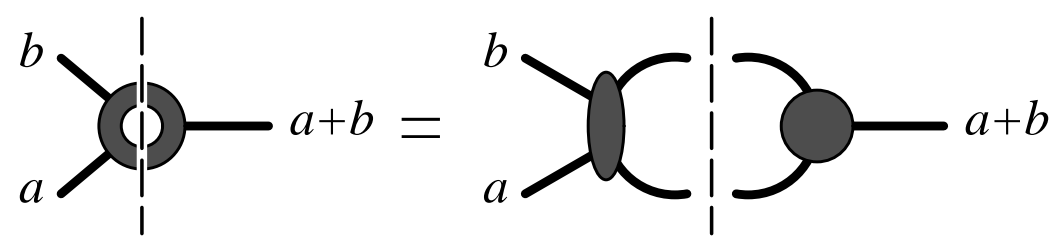

Figure 3. The defining equation for the one-loop splitting amplitude. 
The cut in eqn. (4.1) thus becomes

$$
\begin{aligned}
& \sum_{\substack{\text { ph. pol. } \\
\sigma, \lambda_{1}, \lambda_{2}}} A_{n-1}^{\text {tree }}\left((a+b)^{\sigma}, b+1, \ldots, a-1\right) \\
& \times \int d \operatorname{LIPS}^{4-2 \epsilon}\left(\ell_{1}, \ell_{2}\right) \operatorname{Split}_{-\sigma}^{\mathrm{tree}}\left(-\ell_{2}^{-\lambda_{2}},-\ell_{1}^{-\lambda_{1}}\right) A_{4}^{\mathrm{tree}}\left(\ell_{2}^{\lambda_{2}}, a^{\lambda_{a}}, b^{\lambda_{b}}, \ell_{1}^{\lambda_{1}}\right) \\
& \left.\equiv \sum_{\text {ph. pol. } \sigma} A_{n-1}^{\text {tree }}\left((a+b)^{\sigma}, b, \ldots, a-1\right) \operatorname{Split}_{-\sigma}^{1-\text { loop }}\left(a^{\lambda_{a}}, b^{\lambda_{b}}\right)\right|_{s_{a b} \text { cut }}
\end{aligned}
$$

in the collinear limit. Since this channel is the only one in which Split ${ }^{1-l o o p}$ has a cut, we can immediately write down a formula for it,

$$
\begin{array}{r}
\sum_{\text {ph. pol. } \lambda_{1}, \lambda_{2}}^{\operatorname{Split}_{-\sigma}^{1-\text { loop }}\left(a^{\lambda_{a}}, b^{\lambda_{b}}\right)=} \int \frac{d^{4-2 \epsilon} \ell}{(2 \pi)^{4-2 \epsilon}} \frac{i}{\ell^{2}} \operatorname{Split}_{-\sigma}^{\text {tree }}\left((\ell+a+b)^{-\lambda_{2}},-\ell^{-\lambda_{1}}\right) \frac{i}{\left(\ell+k_{a}+k_{b}\right)^{2}} \\
\times A_{4}^{\text {tree }}\left((-\ell-a-b)^{\lambda_{2}}, a^{\lambda_{a}}, b^{\lambda_{b}}, \ell^{\lambda_{1}}\right)
\end{array}
$$

The restriction to physical polarizations is important; it will give rise to transverse projection operators inside the loop. The defining equation is depicted graphically in fig. 3 .

If we now combine all cuts, we find in agreement with the known result [7], also shown in fig. 2 ,

$$
\begin{aligned}
& A_{n}^{1 \text {-loop }}\left(1, \ldots, a^{\lambda_{a}}, b^{\lambda_{b}}, \ldots, n\right) \stackrel{a \| b}{\longrightarrow} \\
& \quad \sum_{\text {ph. pol. } \sigma}\left(\operatorname{Split}_{-\sigma}^{\text {tree }}\left(a^{\lambda_{a}}, b^{\lambda_{b}}\right) A_{n-1}^{1-\text { loop }}\left(1, \ldots,(a+b)^{\sigma}, \ldots, n\right)\right. \\
& \left.\quad+\operatorname{Split}_{-\sigma}^{1-\text { loop }}\left(a^{\lambda_{a}}, b^{\lambda_{b}}\right) A_{n-1}^{\text {tree }}\left(1, \ldots,(a+b)^{\sigma}, \ldots, n\right)\right) .
\end{aligned}
$$

This completes the proof of the universality of collinear factorization at one loop. Unlike the proof of Bern and Chalmers, it provides a compact formula for computing the one-loop splitting amplitudes. (Explicit computations with this formula will be presented elsewhere [20].) As we shall see in following sections, it also generalizes nicely beyond one-loop amplitudes. 


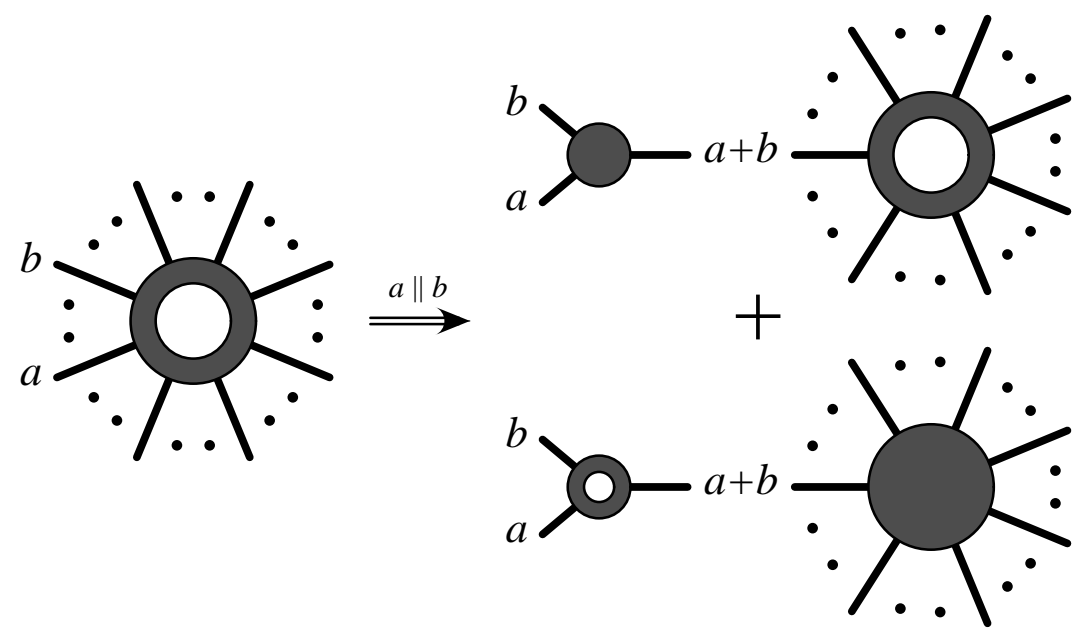

Figure 2. A schematic depiction of the collinear factorization of one-loop amplitudes.

\section{Two-Loop Collinear Factorization}

The analysis in the previous sections extends readily to two-loop amplitudes. At two loops or beyond, leading-color amplitudes (or equivalently, planar color-ordered amplitudes) do not suffice to construct the full amplitude. In the following, I will consider explicitly only leading-color amplitudes, though the analysis can be extended to subleading-color ones.

The basic equation (2.2) of the unitarity-based method has an analog at two- (or higher-) loop order. The sum over cuts now includes not only two-particle cuts but three-particle cuts as well. The amplitudes on either side of the cut are no longer just tree amplitudes, but may be loop amplitudes as well,

$$
\begin{aligned}
& \left.A_{n}^{2-\text { loop }}(1, \ldots, a, b, \ldots, n)\right|_{t_{c \cdots d} \text { cut }}= \\
& \quad \int d \operatorname{LIPS}^{4-2 \epsilon}\left(\ell_{1}, \ell_{2}\right) A_{n-m+2}^{1-\text { loop }}\left(\ell_{1}, c, \ldots, d, \ell_{2}\right) A_{m+2}^{\text {tree }}\left(-\ell_{2}, d+1, \ldots, a, b, \ldots, c-1,-\ell_{1}\right) \\
& \quad+\int d \operatorname{LIPS}^{4-2 \epsilon}\left(\ell_{1}, \ell_{2}\right) A_{n-m+2}^{\text {tree }}\left(\ell_{1}, c, \ldots, d, \ell_{2}\right) A_{m+2}^{1-\text { loop }}\left(-\ell_{2}, d+1, \ldots, a, b, \ldots, c-1,-\ell_{1}\right) \\
& \quad+\int d \operatorname{LIPS}^{4-2 \epsilon}\left(\ell_{1}, \ell_{2}, \ell_{3}\right) A_{n-m+3}^{\text {tree }}\left(\ell_{1}, c, \ldots, d, \ell_{2}, \ell_{3}\right) \\
& \quad \times A_{m+3}^{\text {tree }}\left(-\ell_{3},-\ell_{2}, d+1, \ldots, a, b, \ldots, c-1,-\ell_{1}\right) .
\end{aligned}
$$

Note that in the three-particle cuts, all the $\ell_{i}$ must be adjacent in order to obtain a leading-color (planar) contribution; in the labelling used here, $\ell_{3}$ is actually the middle leg crossing the cut. The sum over intermediate particle types and physical polarizations should again be understood implicitly.

The arguments on the ordering of limits given in section 2 are in fact quite general, and apply to higher-loop amplitudes as well. We may thus take the singular limit first, in all channels other 
than that of the singular invariant $s_{a b}$. To do so, we must use both the tree-level collinear limit (3.2) as well as the one-loop limit (4.5), whereupon we find that the above expression has the following limit,

$$
\begin{aligned}
& \sum_{\text {ph. pol. } \sigma} \operatorname{Split}_{-\sigma}^{\text {tree }}\left(a^{\lambda_{a}}, b^{\lambda_{b}}\right)\left[\int d \operatorname{LIPS}^{4-2 \epsilon}\left(\ell_{1}, \ell_{2}\right) A_{n-m+2}^{1-\text { loop }}\left(\ell_{1}, c, \ldots, d, \ell_{2}\right)\right. \\
& \times A_{m+1}^{\text {tree }}\left(-\ell_{2}, d+1, \ldots,(a+b)^{\sigma}, \ldots, c-1,-\ell_{1}\right) \\
& +\int d \operatorname{LIPS}^{4-2 \epsilon}\left(\ell_{1}, \ell_{2}\right) A_{n-m+2}^{\text {tree }}\left(\ell_{1}, c, \ldots, d, \ell_{2}\right) \\
& \times A_{m+1}^{1-\text { loop }}\left(-\ell_{2}, d+1, \ldots,(a+b)^{\sigma}, \ldots, c-1,-\ell_{1}\right) \\
& +\int d \operatorname{LIPS}^{4-2 \epsilon}\left(\ell_{1}, \ell_{2}, \ell_{3}\right) A_{n-m+3}^{\text {tree }}\left(\ell_{1}, c, \ldots, d, \ell_{2}, \ell_{3}\right) \\
& \left.\times A_{m+2}^{\text {tree }}\left(-\ell_{3},-\ell_{2}, d+1, \ldots,(a+b)^{\sigma}, \ldots, c-1,-\ell_{1}\right)\right] \\
& +\sum_{\text {ph. pol. } \sigma} \operatorname{Split}_{-\sigma}^{1-\text { loop }}\left(a^{\lambda_{a}}, b^{\lambda_{b}}\right) \int d \operatorname{LIPS}^{4-2 \epsilon}\left(\ell_{1}, \ell_{2}\right) A_{n-m+2}^{\text {tree }}\left(\ell_{1}, c, \ldots, d, \ell_{2}\right) \\
& \times A_{m+1}^{\text {tree }}\left(-\ell_{2}, d+1, \ldots,(a+b)^{\sigma}, \ldots, c-1,-\ell_{1}\right) \\
& =\left.\sum_{\text {ph. pol. } \sigma} \operatorname{Split}_{-\sigma}^{\text {tree }}\left(a^{\lambda_{a}}, b^{\lambda_{b}}\right) A_{n-1}^{2-\text { loop }}\left(1, \ldots,(a+b)^{\sigma}, \ldots, n\right)\right|_{t_{c \cdots d} \text { cut }} \\
& +\left.\sum_{\text {ph. pol. } \sigma} \operatorname{Split}_{-\sigma}^{1-\text { loop }}\left(a^{\lambda_{a}}, b^{\lambda_{b}}\right) A_{n-1}^{1-\text { loop }}\left(1, \ldots,(a+b)^{\sigma}, \ldots, n\right)\right|_{t_{c \cdots d} \text { cut }}
\end{aligned}
$$

We must again consider the singular channel specially. The cut in that channel is,

$$
\begin{aligned}
& \left.A_{n}^{2-\text { loop }}(1, \ldots, a, b, \ldots, n)\right|_{s_{a b} \text { cut }}= \\
& \quad \int d \operatorname{LIPS}^{4-2 \epsilon}\left(\ell_{1}, \ell_{2}\right) A_{n}^{1-\text { loop }}\left(-\ell_{1}, b+1, \ldots, a-1,-\ell_{2}\right) A_{4}^{\text {tree }}\left(\ell_{2}, a, b, \ell_{1}\right) \\
& \quad+\int d \operatorname{LIPS}^{4-2 \epsilon}\left(\ell_{1}, \ell_{2}\right) A_{n}^{\text {tree }}\left(-\ell_{1}, b+1, \ldots, a-1,-\ell_{2}\right) A_{4}^{1-\text { loop }}\left(\ell_{2}, a, b, \ell_{1}\right) \\
& \quad+\int d \operatorname{LIPS}^{4-2 \epsilon}\left(\ell_{1}, \ell_{2}, \ell_{3}\right) A_{n+1}^{\text {tree }}\left(-\ell_{1}, b+1, \ldots, a-1,-\ell_{2},-\ell_{3}\right) A_{5}^{\text {tree }}\left(\ell_{3}, \ell_{2}, a, b, \ell_{1}\right)
\end{aligned}
$$

Once again, momentum conservation forces all legs crossing the cut to become collinear. For the two particle cuts, this is just the statement that $s_{\ell_{1} \ell_{2}} \rightarrow 0$, with the $\ell_{i}$ massless. For the threeparticle cuts, the three-particle invariant vanishes, $t_{\ell_{1} \ell_{2} \ell_{3}} \rightarrow 0$. Because the $\ell_{i}$ are physical (in a $D$-dimensional sense), they all have positive energies; the vanishing of the three-particle invariant thus forces the separate two-particle invariants to vanish as well, $s_{\ell_{i} \ell_{j}} \rightarrow 0$.

The behavior of tree amplitudes when three color-adjacent external particles become collinear is governed by analogs of the collinear splitting amplitude,

$$
\begin{aligned}
& A_{n+2}^{\text {tree }}\left(\ldots, a^{\lambda_{a}}, b^{\lambda_{b}}, c^{\lambda_{c}} \ldots\right) \stackrel{k_{a} \cdot k_{b}, k_{a} \cdot k_{c}, k_{b} \cdot k_{c} \rightarrow 0}{\longrightarrow} \\
& \sum_{\text {ph. pol. } \sigma} \operatorname{Split}_{-\sigma}^{\text {tree }}\left(a^{\lambda_{a}}, b^{\lambda_{b}}, c^{\lambda_{c}}\right) A_{n}^{\text {tree }}\left(\ldots,(a+b+c)^{\sigma}, \ldots\right),
\end{aligned}
$$


valid when $n \geq 4$. The squares of such double-collinear splitting amplitudes have been computed by Glover and Campbell [21] and by Catani and Grazzini [22].

As in the one-loop case, we cannot factorize the four- or five-point amplitudes on the right-hand side of the cut. We can, however, factorize the amplitudes on the left-hand side, obtaining

$$
\begin{aligned}
& \sum_{\substack{\text { ph. pol. } \\
\sigma, \lambda_{1}, \lambda_{2}}} A_{n-1}^{1-\text { loop }}\left((a+b)^{\sigma}, b+1, \ldots, a-1\right) \\
& \quad \times \int d \operatorname{LIPS}^{4-2 \epsilon}\left(\ell_{1}, \ell_{2}\right) \operatorname{Split}_{-\sigma}^{\text {tree }}\left(-\ell_{2}^{\lambda_{2}},-\ell_{1}^{\lambda_{1}}\right) A_{4}^{\text {tree }}\left(\ell_{2}, a, b, \ell_{1}\right) \\
& +\sum_{\substack{\text { ph. pol. } \\
\sigma, \lambda_{1}, \lambda_{2}}} A_{n-1}^{\text {tree }}\left((a+b)^{\sigma}, b+1, \ldots, a-1\right) \\
& \quad \times \int d \operatorname{LIPS}^{4-2 \epsilon}\left(\ell_{1}, \ell_{2}\right) \operatorname{Split}_{-\sigma}^{1-\text { loop }}\left(-\ell_{2}^{\lambda_{2}},-\ell_{1}^{\lambda_{1}}\right) A_{4}^{\text {tree }}\left(\ell_{2}, a, b, \ell_{1}\right) \\
& +\sum_{\substack{\text { ph. pol. } \\
\sigma, \lambda_{1}, \lambda_{2}}} A_{n-1}^{\text {tree }}\left((a+b)^{\sigma}, b+1, \ldots, a-1\right) \\
& \quad \times \int d \operatorname{LIPS}^{4-2 \epsilon}\left(\ell_{1}, \ell_{2}\right) \operatorname{Split}_{-\sigma}^{\text {tree }}\left(-\ell_{2}^{\lambda_{2}},-\ell_{1}^{\lambda_{1}}\right) A_{4}^{1-\text { loop }}\left(\ell_{2}, a, b, \ell_{1}\right) \\
& +\sum_{\substack{\text { ph. pol. } \\
\sigma, \lambda_{1}, \lambda_{2}, \lambda_{3}}} A_{n-1}^{\text {tree }}\left((a+b)^{\sigma}, b+1, \ldots, a-1\right) \\
& \quad \times \int d \operatorname{LIPS}^{4-2 \epsilon}\left(\ell_{1}, \ell_{2}, \ell_{3}\right) \operatorname{Split}_{-\sigma}^{\text {tree }}\left(-\ell_{2}^{\lambda_{2}},-\ell_{3}^{\lambda_{3}},-\ell_{1}^{\lambda_{1}}\right) A_{5}^{\text {tree }}\left(\ell_{3}, \ell_{2}, a, b, \ell_{1}\right) .
\end{aligned}
$$

We recognize

$$
\int d \operatorname{LIPS}^{4-2 \epsilon}\left(\ell_{1}, \ell_{2}\right) \operatorname{Split}_{-\sigma}^{\mathrm{tree}}\left(-\ell_{2}^{\lambda_{2}},-\ell_{1}^{\lambda_{1}}\right) A_{4}^{\text {tree }}\left(\ell_{2}, a, b, \ell_{1}\right)
$$

as the cut of the one-loop splitting function, so that eqn. (5.3) can be written as

$$
\begin{aligned}
& \left.\sum_{\substack{\text { ph. pol. } \\
\sigma, \lambda_{1}, \lambda_{2}}} \operatorname{Split}_{-\sigma}^{1-\text { loop }}\left(a^{\lambda_{a}}, b^{\lambda_{b}}\right)\right|_{s_{a b} \text { cut }} A_{n-1}^{1-\text { loop }}\left((a+b)^{\sigma}, b+1, \ldots, a-1\right) \\
& \quad+\left.\sum_{\substack{\text { ph. pol. } \\
\sigma, \lambda_{1}, \lambda_{2}}} \operatorname{Split}_{-\sigma}^{2-\text { loop }}\left(a^{\lambda_{a}}, b^{\lambda_{b}}\right)\right|_{s_{a b} \text { cut }} A_{n-1}^{\text {tree }}\left((a+b)^{\sigma}, b+1, \ldots, a-1\right),
\end{aligned}
$$

where I have introduced a new quantity, the two-loop splitting amplitude Split ${ }^{2-l o o p}$. It is defined by its cuts,

$$
\begin{aligned}
\operatorname{Split}_{-\sigma}^{2-\text { loop }} & \left.\left(a^{\lambda_{a}}, b^{\lambda_{b}}\right)\right|_{s_{a b} \text { cut }} \\
= & \sum_{\substack{\text { ph. pol. } \\
\sigma, \lambda_{1}, \lambda_{2}}} \int d \operatorname{LIPS}^{4-2 \epsilon}\left(\ell_{1}, \ell_{2}\right)\left[\operatorname{Split}_{-\sigma}^{1-\text { loop }}\left(-\ell_{2}^{\lambda_{2}},-\ell_{1}^{\lambda_{1}}\right) A_{4}^{\text {tree }}\left(\ell_{2}, a, b, \ell_{1}\right)\right. \\
& \left.\quad+\operatorname{Split}_{-\sigma}^{\text {tree }}\left(-\ell_{2}^{\lambda_{2}},-\ell_{1}^{\lambda_{1}}\right) A_{4}^{1-\text { loop }}\left(\ell_{2}, a, b, \ell_{1}\right)\right] \\
& +\sum_{\substack{\text { ph. pol. } \\
\sigma, \lambda_{1}, \lambda_{2}, \lambda_{3}}} \int d \operatorname{LIPS}^{4-2 \epsilon}\left(\ell_{1}, \ell_{2}, \ell_{3}\right) \operatorname{Split}_{-\sigma}^{\text {tree }}\left(-\ell_{2}^{\lambda_{2}},-\ell_{3}^{\lambda_{3}},-\ell_{1}^{\lambda_{1}}\right) A_{5}^{\text {tree }}\left(\ell_{3}, \ell_{2}, a, b, \ell_{1}\right) .
\end{aligned}
$$


The splitting amplitude has no cuts in other channels. Because the cut in this channel is taken to all orders in $\epsilon$, this equation suffices to reconstruct it completely.
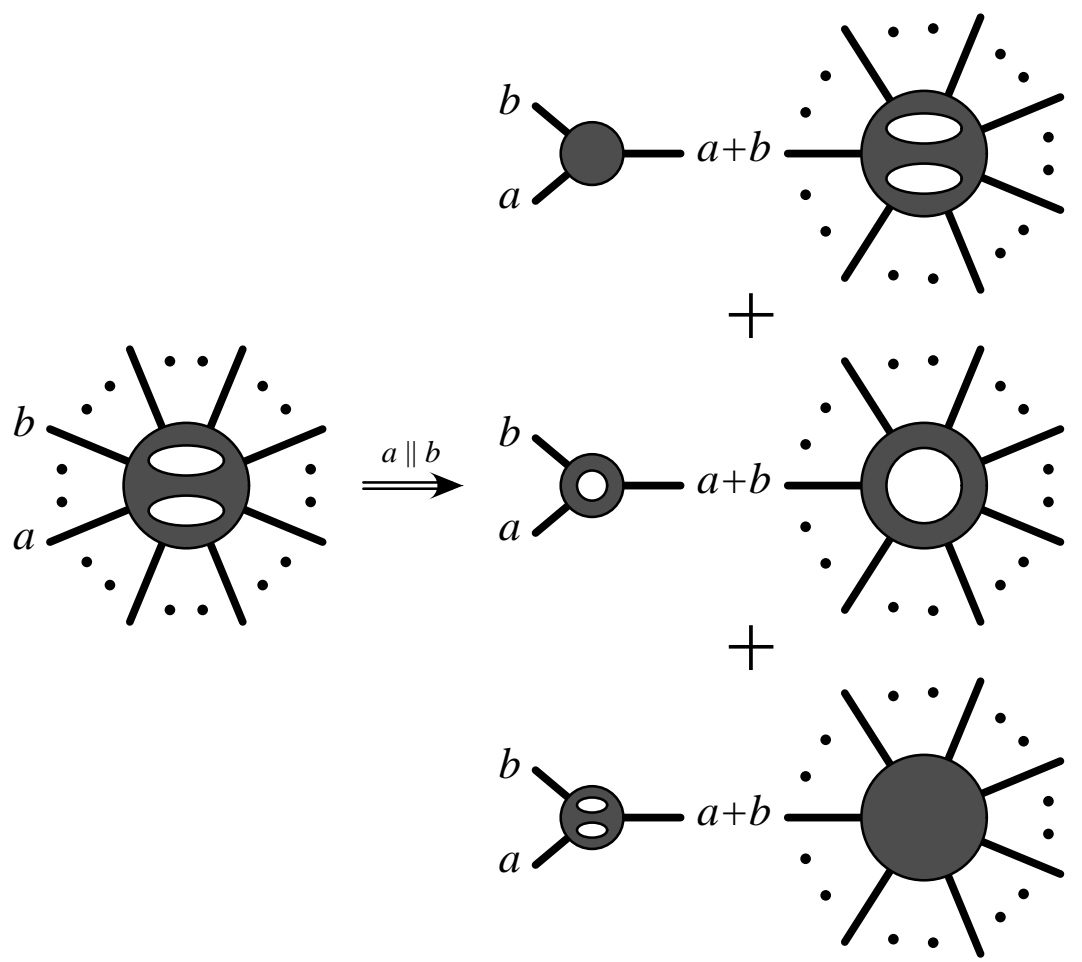

Figure 5. A schematic depiction of the collinear factorization of two-loop amplitudes.

The first term in eqn. (5.7) repoduces the second term on the last line of eqn. (5.2); combining all channels, we finally obtain the collinear behavior of a leading-color two-loop amplitude, also shown schematically in fig. 5 ,

$$
\begin{aligned}
A_{n}^{2-\operatorname{loop}}\left(1, \ldots, a^{\lambda_{a}}, b^{\lambda_{b}}, \ldots, n\right) \stackrel{k_{a} \cdot k_{b} \rightarrow 0}{\longrightarrow} & \sum_{\text {ph. pol. } \sigma}[ \\
& \operatorname{Split}_{-\sigma}^{\text {tree }}\left(a^{\lambda_{a}}, b^{\lambda_{b}}\right) A_{n-1}^{2-\text { loop }}\left(1, \ldots,(a+b)^{\sigma}, \ldots, n\right) \\
& +\operatorname{Split}_{-\sigma}^{1-\text { loop }}\left(a^{\lambda_{a}}, b^{\lambda_{b}}\right) A_{n-1}^{1-\text { loop }}\left(1, \ldots,(a+b)^{\sigma}, \ldots, n\right) \\
& \left.+\operatorname{Split}_{-\sigma}^{2-\text { loop }}\left(a^{\lambda_{a}}, b^{\lambda_{b}}\right) A_{n-1}^{\text {tree }}\left(1, \ldots,(a+b)^{\sigma}, \ldots, n\right)\right] .
\end{aligned}
$$

\section{One-Loop Corrections to the Double-Collinear Splitting Amplitude}

As three external legs become simultaneously collinear in a one-loop amplitude, we expect a factorization analogous to that at tree level, eqn. (5.4). We can derive it using an approach similar 
to that in previous sections. Much of the discussion will carry over, but there are certain differences worthy of closer examination.

The major difference is that the double-collinear splitting amplitude is no longer a function of a sole invariant; rather, it depends on the two neighboring two-particle invariants $s_{a b}$ and $s_{b c}$, as well as the overall three-particle invariant $t_{a b c}$. All of these invariants vanish in the limit. (I take the order of the external legs to be $a, b, c$.) As discussed by Campbell and Glover [21], the tree-level double-collinear splitting amplitude contains all terms that are singular in two of the invariants. (Terms singular in only one invariant would eventually give rise to a vanishing contribution to physical processes.) At one loop, we will acquire additional epsilonic powers of the invariants (and possibly more complicated analytic functions of their ratios), but the basic requirement of having integral or half-integral power singularities in two of the invariants remains.

As in the simple splitting amplitudes, these integral powers can arise either from amplitudes on either side of the cut, or else from the loop integral whose cut we are taking. In the former case, all three of the collinear legs must be on the same side of the cut. In the latter case, an inverse power in a given invariant also implies the integral function must contain a cut in that invariant. Let us focus on the inverse powers of $t_{a b c}$. Two of the possible pole combinations - in $\left(s_{a b}, t_{a b c}\right)$ and in $\left(s_{b c}, t_{a b c}\right)$ - manifestly require that the loop integral have a cut in $t_{a b c}$. The third combination $\left(s_{a b}, s_{b c}\right)$ also requires such a cut. To see this, note that it must have cuts in both $s_{a b}$ and $s_{b c}$, and thus must have uncancelled propagators between legs $a-1$ and $a$, and likewise $c$ and $c+1$. These two uncancelled propagators force the integral to have a cut in the three-particle channel as well.

The set of cuts we must consider is thus exactly analogous to that for the simple collinear splitting amplitude: all legs must be on one side of the cut. Contributions missed by ignoring cuts that separate the collinear legs will be be picked up in the primary singular channel, where the three legs will sit alone on one side of the cut. The remaining arguments about the order of limits go through as before: in channels other than the singular channel, we can take the singular limit before performing the cut, in the singular channel we must tread carefully. (These arguments also carry over to general multi-collinear splitting amplitudes.)

In a non-singular channel, the limit of eqn. (3.1) again produces a tree-level splitting amplitude,

$$
\begin{aligned}
& \left.A_{n}^{1-\text { loop }}(1, \ldots, a, b, c, \ldots, n)\right|_{t_{d \cdots f} \text { cut }} k_{a} \cdot k_{b}, k_{a} \cdot k_{c}, k_{b} \cdot k_{c} \rightarrow 0 \\
& \sum_{\text {ph. pol. } \sigma} \operatorname{Split}_{-\sigma}^{\text {tree }}\left(a^{\lambda_{a}}, b^{\lambda_{b}}, c^{\lambda_{c}}\right) \int d \operatorname{LIPS}^{4-2 \epsilon}\left(\ell_{1},-\ell_{2}\right) \\
& \times A_{n-m+2}^{\text {tree }}\left(\ell_{1}, d, \ldots, f,-\ell_{2}\right) A_{m}^{\text {tree }}\left(\ell_{2}, f+1, \ldots,(a+b+c)^{\sigma}, \ldots, d-1,-\ell_{1}\right) \\
& =\left.\sum_{\text {ph. pol. } \sigma} \operatorname{Split}_{-\sigma}^{\text {tree }}\left(a^{\lambda_{a}}, b^{\lambda_{b}}, c^{\lambda_{c}}\right) A_{n-2}^{1-\operatorname{loop}}\left(1, \ldots,(a+b+c)^{\sigma}, \ldots, n\right)\right|_{t_{d \cdots f} \text { cut }} .
\end{aligned}
$$


In the three-particle singular channel, the cut is

$$
\begin{aligned}
& \left.A_{n}^{1-\operatorname{loop}}(1, \ldots, a, b, c, \ldots, n)\right|_{t_{a b c} \text { cut }}= \\
& \quad \sum_{\text {ph. pol. } \lambda_{1,2}} \int d \operatorname{LIPS}^{4-2 \epsilon}\left(\ell_{1}, \ell_{2}\right) A_{n-1}^{\text {tree }}\left(-\ell_{1}^{-\lambda_{1}}, c+1, \ldots, a-1,-\ell_{2}^{-\lambda_{2}}\right) A_{5}^{\text {tree }}\left(\ell_{2}^{\lambda_{2}}, a, b, c, \ell_{1}^{\lambda_{1}}\right) .
\end{aligned}
$$

The vanishing of the three-particle invariant forces the vanishing of the invariant of the legs crossing the cut, $s_{\ell_{1} \ell_{2}}$, and we can again use this to factorize the amplitude on the left-hand side, so the cut becomes

$$
\begin{aligned}
& \sum_{\substack{\text { ph. pol. } \\
\sigma, \lambda_{1}, \lambda_{2}}} A_{n-2}^{\text {tree }}\left((a+b+c)^{\sigma}, c+1, \ldots, a-1\right) \\
& \quad \times \int d \operatorname{LIPS}^{4-2 \epsilon}\left(\ell_{1}, \ell_{2}\right) \operatorname{Split}_{-\sigma}^{\text {tree }}\left(-\ell_{2}^{-\lambda_{2}},-\ell_{1}^{-\lambda_{1}}\right) A_{5}^{\text {tree }}\left(\ell_{2}^{\lambda_{2}}, a^{\lambda_{a}}, b^{\lambda_{b}}, c^{\lambda_{c}}, \ell_{1}^{\lambda_{1}}\right) \\
& \left.\equiv \sum_{\text {ph. pol. } \sigma} A_{n-2}^{\text {tree }}\left((a+b+c)^{\sigma}, c+1, \ldots, a-1\right) \operatorname{Split}_{-\sigma}^{1-\text { loop }}\left(a^{\lambda_{a}}, b^{\lambda_{b}}, c^{\lambda_{b}}\right)\right|_{t_{a b c} \text { cut }},
\end{aligned}
$$

where the latter equation defines the one-loop double splitting amplitude.

Although this splitting amplitude will also have cuts in the two-particle invariants within the collinear set, as discussed above it must have a cut in the three-particle channel, and hence this cut suffices to reconstruct it fully,

$$
\begin{aligned}
& \operatorname{Split}_{-\sigma}^{1-\text { loop }}\left(a^{\lambda_{a}}, b^{\lambda_{b}}, c^{\lambda_{c}}\right)= \\
& \qquad \begin{array}{l}
\sum_{\text {ph. pol. } \lambda_{1}, \lambda_{2}} \int \frac{d^{4-2 \epsilon} \ell}{(2 \pi)^{4-2 \epsilon}} \frac{i}{\ell^{2}} \\
\operatorname{Split}_{-\sigma}^{\text {tree }}\left((\ell+a+b+c)^{-\lambda_{2}},-\ell^{-\lambda_{1}}\right) \frac{i}{\left(\ell+k_{a}+k_{b}+k_{c}\right)^{2}} \\
\times A_{5}^{\text {tree }}\left((-\ell-a-b)^{\lambda_{2}}, a^{\lambda_{a}}, b^{\lambda_{b}}, c^{\lambda_{c}}, \ell^{\lambda_{1}}\right)
\end{array}
\end{aligned}
$$

The restriction to physical polarizations will once again give rise to transverse projection operators inside the loop.

Combining all cuts, we find a double-collinear factorization formula very similar to the simple collinear one,

$$
\begin{aligned}
& A_{n}^{1 \text {-loop }}\left(1, \ldots, a^{\lambda_{a}}, b^{\lambda_{b}}, c^{\lambda_{c}} \ldots, n\right) \stackrel{a\|b\| c}{\longrightarrow} \\
& \quad \sum_{\text {ph. pol. } \sigma}\left(\operatorname{Split}_{-\sigma}^{\text {tree }}\left(a^{\lambda_{a}}, b^{\lambda_{b}}, c^{\lambda_{c}}\right) A_{n-2}^{1-\text { loop }}\left(1, \ldots,(a+b+c)^{\sigma}, \ldots, n\right)\right. \\
& \left.\quad+\operatorname{Split}_{-\sigma}^{1-\text { loop }}\left(a^{\lambda_{a}}, b^{\lambda_{b}}, c^{\lambda_{c}}\right) A_{n-2}^{\text {tree }}\left(1, \ldots,(a+b+c)^{\sigma}, \ldots, n\right)\right) .
\end{aligned}
$$

\section{Collinear Factorization to All Orders}

In this section, I give a proof of collinear factorization to all orders, generalizing further the results of the previous sections. As in the two-loop case, I will restrict attention to leading-color amplitudes, that is to coefficients of $N^{l}$ times the tree-level color structure in an $l$-loop amplitude. 
The general cut formula here is

$$
\begin{aligned}
& \left.A_{n}^{l \text {-loop }}\left(1, \ldots, a_{1}, a_{2}, \ldots, a_{c}, \ldots, n\right)\right|_{t_{d \ldots f} \text { cut }}= \\
& \quad \sum_{j=2}^{l+1} \int d \operatorname{LIPS}^{4-2 \epsilon}\left(\ell_{1}, \ldots, \ell_{j}\right) \\
& \quad \times \sum_{k=0}^{l+1-j} A_{n-m+j}^{k \text {-loop }}\left(d, \ldots, f, \ell_{1}, \ldots, \ell_{j}\right) A_{m+j}^{(l+1-j-k) \text {-loop }}\left(f+1, \ldots, a, b, \ldots, d-1,-\ell_{j}, \ldots,-\ell_{1}\right)
\end{aligned}
$$

so long as the $a_{i}$ all end up on one side of the cut. (As in earlier sections, we can ignore contributions where different $a_{i}$ end up on opposite sides of the cut.) The outer sum is on $j$-particle cuts, the inner one on the different $j$-particle cuts; $m$ is as in previous sections, the number of external momenta on the far side of the cut; and '0-loop' means 'tree'. The sum over intermediate particle types and physical polarizations is, as always, implicit.

The proof will proceed by induction, in fact a double induction, over both the number of loops and the number of color-adjacent legs becoming collinear simultaneously. Denote by $S_{c}$ the set of invariants built out of consecutive momenta in $\left\{a_{1}, \ldots, a_{c}\right\}$, and indicate the multiple collinear limit by $S_{c} \rightarrow 0$ (that is, all of the invariants are supposed to vanish).

Now assume that for $r<l$ and $c<n-2$, that we have the following factorization,

$$
\begin{aligned}
& A_{n}^{r \text {-loop }}\left(1, \ldots, a_{1}^{\lambda_{1}}, a_{2}^{\lambda_{2}}, \ldots, a_{c}^{\lambda_{c}}, \ldots, n\right) \stackrel{S_{c} \rightarrow 0}{\longrightarrow} \\
& \quad \sum_{\text {ph. pol. } \sigma} \sum_{\sigma=0}^{r} \operatorname{Split}_{-\sigma}^{v \text {-loop }}\left(a_{1}^{\lambda_{1}}, a_{2}^{\lambda_{2}}, \ldots, a_{c}^{\lambda_{c}}\right) A_{n-c+1}^{(r-v) \text {-loop }}\left(1, \ldots,\left(a_{1}+\ldots+a_{c}\right)^{\sigma}, \ldots, n\right),
\end{aligned}
$$

The starting point for the induction $-r=1$ and $c=2-$ is the one-loop factorization proven in section 4. In addition, I will make use of the tree-level multi-collinear analog to eqn. (5.4), which can again be derived either from the Berends-Giele recurrence relation or from the string representation.

In all cuts except that in the primary singular channel $\left(K_{c}^{2}=\left(k_{a_{1}}+\ldots+k_{a_{c}}\right)^{2}\right)$, we then find using the above assumption, that

$$
\begin{aligned}
& \left.A_{n}^{l \text {-loop }}\left(1, \ldots, a_{1}^{\lambda_{1}}, a_{2}^{\lambda_{2}}, \ldots, a_{c}^{\lambda_{c}}, \ldots, n\right)\right|_{t_{d \ldots f} \text { cut }} \stackrel{S_{c} \rightarrow 0}{\longrightarrow} \\
& \sum_{j=2}^{l+1} \int d \operatorname{LIPS}^{4-2 \epsilon}\left(\ell_{1}, \ldots, \ell_{j}\right) \sum_{k=0}^{l+1-j} A_{n-m+j}^{k-\text { loop }}\left(d, \ldots, f, \ell_{1}, \ldots, \ell_{j}\right) \\
& \quad \times \sum_{\text {ph. pol. } \sigma} \sum_{v=0}^{l+1-j-k} \operatorname{Split}_{-\sigma}^{v-\text { loop }}\left(a_{1}^{\lambda_{1}}, a_{2}^{\lambda_{2}}, \ldots, a_{c}^{\lambda_{c}}\right) \\
& \quad \times A_{m+j-c+1}^{(l+1-j-k-v) \text { loop }}\left(f+1, \ldots,\left(a_{1}+\ldots+a_{c}\right)^{\sigma}, \ldots, d-1,-\ell_{j}, \ldots,-\ell_{1}\right) .
\end{aligned}
$$


Interchanging the summations,

$$
\sum_{j=2}^{l+1} \sum_{k=0}^{l+1-j} \sum_{v=0}^{l+1-j-k}=\sum_{j=2}^{l+1} \sum_{v=0}^{l+1-j} \sum_{k=0}^{l+1-j-v}=\sum_{v=0}^{l-1} \sum_{j=2}^{l+1-v} \sum_{k=0}^{l+1-j-v},
$$

we can transform eqn. (7.3) to obtain

$$
\begin{aligned}
& \left.A_{n}^{l \text {-loop }}\left(1, \ldots, a_{1}^{\lambda_{1}}, a_{2}^{\lambda_{2}}, \ldots, a_{c}^{\lambda_{c}}, \ldots, n\right)\right|_{t_{d} \ldots f} \stackrel{S_{c} \rightarrow 0}{\longrightarrow} \\
& \quad \sum_{\text {ph. pol. } \sigma} \sum_{v=0}^{l-1} \operatorname{Split}_{-\sigma}^{v \text {-loop }}\left(a_{1}^{\lambda_{1}}, a_{2}^{\lambda_{2}}, \ldots, a_{c}^{\lambda_{c}}\right) \sum_{j=2}^{l-v+1} \int d \operatorname{LIPS}^{4-2 \epsilon}\left(\ell_{1}, \ldots, \ell_{j}\right) \\
& \quad \times \sum_{k=0}^{l-v+1-j} A_{n-m+j}^{k-\text { loop }}\left(d, \ldots, f, \ell_{1}, \ldots, \ell_{j}\right) \\
& \quad \times A_{m+j-c+1}^{(l+1-j-k-v) \text {-loop }}\left(f+1, \ldots,\left(a_{1}+\ldots+a_{c}\right)^{\sigma}, \ldots, d-1,-\ell_{j}, \ldots,-\ell_{1}\right) \\
& =\left.\sum_{\text {ph. pol. } \sigma} \sum_{v=0}^{l-1} \operatorname{Split}_{-\sigma}^{v-\text { loop }}\left(a_{1}^{\lambda_{1}}, a_{2}^{\lambda_{2}}, \ldots, a_{c}^{\lambda_{c}}\right) A_{n-c+1}^{(l-v) \text {-loop }}\left(1, \ldots,\left(a_{1}+\ldots+a_{c}\right)^{\sigma}, \ldots, n\right)\right|_{t_{d \cdots f} \text { cut }}
\end{aligned}
$$

which is exactly eqn. (7.2) in this channel, but now for $r=l$. It again holds for all $c<n-2$.

In the primary singular channel, we obtain in the limit,

$$
\begin{aligned}
& \sum_{j=2}^{l+1} \int d \operatorname{LIPS}^{4-2 \epsilon}\left(\ell_{1}, \ldots, \ell_{j}\right) \sum_{k=0}^{l+1-j} \sum_{\text {ph. pol. }} \sum_{v=0}^{k} \operatorname{Split}_{-\sigma}^{v-\text { loop }}\left(\ell_{1}, \ldots, \ell_{j}\right) \\
& \times A_{n-c+1}^{(k-v) \text {-loop }}\left(a_{c}+1, \ldots, a_{1}-1,\left(a_{1}+\ldots+a_{c}\right)^{\sigma}\right) \\
& \times A_{c+j}^{(l+1-j-k) \text {-loop }}\left(a_{1}^{\lambda_{1}}, a_{2}^{\lambda_{2}}, \ldots, a_{c}^{\lambda_{c}},-\ell_{j}, \ldots,-\ell_{1}\right) \\
&=\sum_{k=0}^{l-1} \sum_{\text {ph. pol. } \sigma} \sum_{v=0}^{k} A_{n-c+1}^{(k-v) \text {-loop }}\left(a_{c}+1, \ldots, a_{1}-1,\left(a-1+\ldots+a_{c}\right)^{\sigma}\right) \\
& \quad \times \sum_{j=2}^{l+1-k} \int d \operatorname{LIPS}^{4-2 \epsilon}\left(\ell_{1}, \ldots, \ell_{j}\right) \operatorname{Split}_{-\sigma}^{v \text {-loop }}\left(\ell_{1}, \ldots, \ell_{j}\right) \\
& \times A_{c+j}^{(l+1-j-k) \text {-loop }}\left(a_{1}^{\lambda_{1}}, a_{2}^{\lambda_{2}}, \ldots, a_{c}^{\lambda_{c}},-\ell_{j}, \ldots,-\ell_{1}\right)
\end{aligned}
$$

which gives us an explicit formula allowing the reconstruction of the $l$-loop multiparticle splitting amplitude in eqn. (7.2) as the coefficient of $A_{n-c+1}^{\text {tree }}$,

$$
\begin{aligned}
& \left.\operatorname{Split}_{-\sigma}^{l-\text { loop }}\left(a_{1}^{\lambda_{1}}, a_{2}^{\lambda_{2}}, \ldots, a_{c}^{\lambda_{c}}\right)\right|_{K_{c}^{2} \text { cut }} \\
& =\sum_{k=0}^{l-1} \sum_{j=2}^{l+1-k} \sum_{\text {ph. pol. } \sigma_{i}} \int d \operatorname{LIPS}^{4-2 \epsilon}\left(\ell_{1}, \ldots, \ell_{j}\right) \operatorname{Split}_{-\sigma}^{k-\text { loop }}\left(\ell_{1}^{-\sigma_{1}}, \ldots, \ell_{j}^{-\sigma_{j}}\right) \\
& \times A_{c+j}^{(l+1-j-k) \text {-loop }}\left(a_{1}^{\lambda_{1}}, a_{2}^{\lambda_{2}}, \ldots, a_{c}^{\lambda_{c}},-\ell_{j}^{\sigma_{j}}, \ldots,-\ell_{1}^{\sigma_{1}}\right)
\end{aligned}
$$

Combining the two kinds of channels, we obtain eqn. (7.2) uniformly for $r=l$, thereby proving the desired result. 


\section{Conclusions}

The square-root factorization of amplitudes in the collinear limit is a striking feature of gauge theories, yet one that hides subtleties. The subtleties, and the complexity of a conventional diagrammatic approach to the problem, are associated with the presence of infrared singularities. The naive intuition of computing only those diagrams associated, for example, with fig. 2, is not quite right. This is demonstrated graphically by the presence of dilogarithms in the quark-gluon splitting amplitudes [7,8], which cannot arise from massless three-point integrals at one loop. The unitarity-based proof given in this paper circumvents these complexities, and gives as well explicit formulæ for computing the splitting amplitudes.

I thank P. Uwer, Z. Bern, and L. Dixon for helpful comments. 


\section{References}

[1] G. Altarelli and G. Parisi, Nucl. Phys. B126:298 (1977).

[2] A. Bassetto, M. Ciafaloni, and G. Marchesini, Phys. Rep. 100:201 (1983).

[3] W.T. Giele and E.W.N. Glover, Phys. Rev. D46:1980 (1992).

[4] S. Frixione, Z. Kunszt, and A. Signer, Nucl. Phys. B467:399 (1996) hep-ph/9512328.

[5] S. Catani and M. Seymour, Phys. Lett. B378:287 (1996) hep-ph/9602277; Nucl. Phys. B485:291 (1997) hep-ph/9605323.

[6] W.T. Giele, E. W. N. Glover and D. A. Kosower, Nucl. Phys. B403:633 (1993) hep-ph/9302225.

[7] Z. Bern, L. Dixon, D. C. Dunbar, and D. A. Kosower, Nucl. Phys. B425:217 (1994) hep$\mathrm{ph} / 9403226$.

[8] Z. Bern, L. Dixon, and D. A. Kosower, Nucl. Phys. B437:259 (1995) hep-ph/9409393.

[9] Z. Bern, L. Dixon, D. C. Dunbar and D. A. Kosower, Nucl. Phys. B435:59 (1995) hep$\mathrm{ph} / 9409265$.

[10] Z. Bern, L. Dixon, and D. A. Kosower, Ann. Rev. Nucl. Part. Sci. 46109 (1996) hep$\mathrm{ph} / 9602280$.

[11] Z. Bern and G. Chalmers, Nucl. Phys. B447:465 (1995) hep-ph/9503236.

[12] F. A. Berends and W. T. Giele, Nucl. Phys. B294:700 (1987);

D. A. Kosower, B.-H. Lee and V. P. Nair, Phys. Lett. 201B:85 (1988);

M. Mangano, S. Parke and Z. Xu, Nucl. Phys. B298:653 (1988);

Z. Bern and D. A. Kosower, Nucl. Phys. B362:389 (1991).

[13] Z. Bern and D. A. Kosower, Nucl. Phys. B379:451 (1992).

[14] S. Catani, M. H. Seymour, and Z. Trócsányi, Phys. Rev. D55:6819 (1997) hep-ph/9610553.

[15] J. C. Collins, Renormalization (Cambridge University Press, 1984).

[16] Z. Bern, L. Dixon, and D. A. Kosower, Nucl. Phys. B412:751 (1994) hep-ph/9306240.

[17] F. A. Berends and W. T. Giele, Nucl. Phys. B306:759 (1988);

D. A. Kosower, Nucl. Phys. B335:23 (1990).

[18] M. Mangano and S.J. Parke, Phys. Rep. 200:301 (1991).

[19] Z. Koba and H. B. Nielsen, Nucl. Phys. B10:633 (1969);

J. H. Schwarz, Phys. Rep. 89:223 (1982).

[20] D. A. Kosower and P. Uwer, in preparation.

[21] J. M. Campbell and E. W. N. Glover, Nucl. Phys. B527:264 (1998) hep-ph/9710255.

[22] S. Catani and M. Grazzini, preprint hep-ph/9810389. 